\title{
End-to-End Outage Probability Analysis for Multi-Source Multi-Relay Systems
}

\author{
Jiguang $\mathrm{He}^{\dagger}$, Iqbal Hussain ${ }^{\dagger}$, Markku Juntti ${ }^{\dagger}$, Tad Matsumoto ${ }^{\dagger \star}$ \\ ${ }^{\dagger}$ Centre for Wireless Communications, FI-90014, University of Oulu, Finland \\ ${ }^{\star}$ Japan Advanced Institute of Science and Technology (JAIST) 1-1 Asahidai, Nomi, Ishikawa, 923-1292, Japan \\ Email: \{jhe, iqbal.hussain, markku.juntti\}@ee.oulu.fi, matumoto@jaist.ac.jp
}

\begin{abstract}
We investigate the end-to-end outage probability over a multi-source multi-relay network assuming that all the wireless links are suffering from independent block fading variations. The overall transmission is divided into two tandem hops. The first hop represents the transmission between sources and relays, while the relays transmit to the destinations in the second hop. We consider specifically a two-source two-relay single-destination network. Based on the decoding process at the relays, the data recovery at the relays can be classified into three cases based on achievable bounded rate regions. The first case where both relays cannot recover both users' data can be characterized as chief executive officer problem at the second hop. For the second case, the relays recover the data of both users, and the outage probability of the second hop can be computed by exploiting maximum ratio transmission. Finally, for the third case, only one of the users' data is recovered by one of the relays, and the outage probability of the second hop can be calculated by using the theorem of source coding with helper. Numerical results closely follow the theoretical analysis and confirm the improved performance of the multiple-antenna relaying over the single-antenna relaying.
\end{abstract}

Index Terms-Outage probability, maximum ratio transmission (MRT), multiple access channel (MAC), multi-source multirelay systems, source coding with helper.

\section{INTRODUCTION}

The concept of lossy decode-and-forward (DF) relaying has been intensively investigated [?], [?], [?]. It provides a promising technique for the future wireless sensor/mesh networks and vehicle-to-vehicle communications, for example. Unlike the traditional lossless DF relaying [?], [?], the lossy DF relaying always forwards the decoded data (including error-free decoded data as well) to the next hop/transmission. The potential performance improvement of the lossy DF relaying as compared to conventional lossless DF relaying was demonstrated in [?].

Most of the conventional information-theoretic analysis was performed for simplified relaying topologies and under the assumption of orthogonal transmission. Meanwhile, less research attention was directed towards nonorthogonal multiple access channels (MACs), especially from the perspective of theoretical analysis of outage probability and practical

This work has been performed in the framework of the FP7 project ICT-619555 RESCUE (Links on-the-fly Technology for Robust, Efficient and Smart Communication in Unpredictable Environments), which is partly funded by the European Union. This work is also partially supported by the network compression based wireless cooperative communication systems (NETCOBRA, No. 268209) project, funded by the Academy of Finland. capacity-approaching code design [?], [?], [?]. Therefore, to address these problems, we consider a multi-source multi-relay single-destination network. In addition, we suppose that the multiple sources simultaneously communicate with the relays, i.e., nonorthogonal transmission for the source-to-relay links. Both single-antenna and multiple-antenna relaying systems are investigated and compared.

It is a challenging task to design practical codes for the nonorthogonal MAC channels that achieve the outer bound of the rate region. Even though the Kasami codes [?] perform very well over the nonorthogonal MAC channels, they cannot achieve the optimal point on the outer bound of the rate region. To overcome this problem, multiple antennas are deployed at the relay nodes, which can convert the MAC problem into a point-to-point transmission problem via zero forcing (ZF)/minimum mean square error (MMSE) filtering [?] at the relay nodes. As a result, practical codes can easily be designed. The capacity-approaching codes, such as turbo codes and low-density parity-check (LDPC) codes, for the point-to-point transmission, can be chosen for multiple-antenna nonorthogonal MAC channels while still keeping their optimality. Moreover, we demonstrate that the multiple-antenna relaying system outperforms the single-antenna relaying system with respect to end-to-end outage performance. The theoretical analysis is verified by numerical examples provided in Section IV.

The rest of the paper is organized as follows. Section II describes the system model and transmission process. Section III details the achievable rate regions of the two hops and outage probabilities of single-antenna and multiple-antenna relaying systems. Numerical results are presented in Section IV. Concluding remarks and future work are given in Section V.

\section{System Model}

For the purpose of notational simplicity, we consider a simple structure composed of two source nodes, two relay nodes, and one destination, shown in Fig. 1. The overall transmission consists of two transmission hops, i.e., sourceto-relay transmission and relay-to-destination transmission. All the transmission links are supposed to be independent and identically distributed (i.i.d.) static frequency-flat block fading. At the first hop, the source nodes transmit independent binary source sequences to the relay nodes simultaneously 


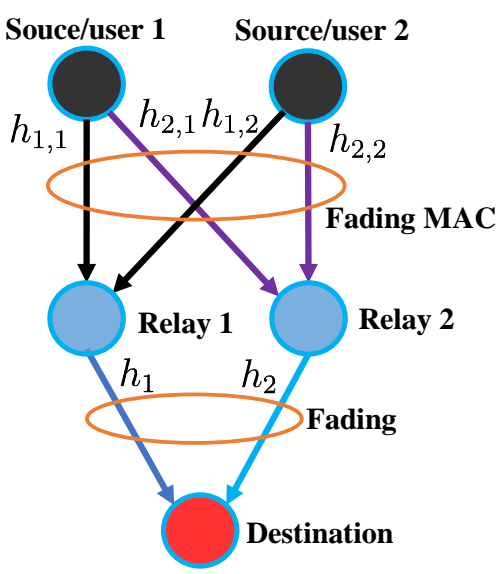

Fig. 1: System model consisting of two sources, two relays, and a single destination.

(two nonorthogonal MACs), and the received signal at the relay nodes can be written as ${ }^{1}$

$$
y_{R_{1}}=\sqrt{P_{1}} h_{1,1} s_{1}+\sqrt{P_{2}} h_{1,2} s_{2}+n_{R_{1}},
$$

and

$$
y_{R_{2}}=\sqrt{P_{1}} h_{2,1} s_{1}+\sqrt{P_{2}} h_{2,2} s_{2}+n_{R_{2}},
$$

where $s_{1}$ and $s_{2}$ are modulated symbols from the codewords ${ }^{2}$ of two independent binary uniform sources $u_{i}$ (originated from user $i$, for $i \in\{1,2\})$ with $\operatorname{Pr}\left(u_{i}=0\right)=\operatorname{Pr}\left(u_{i}=1\right)=0.5$ and $E\left\{\left|s_{i}\right|^{2}\right\}=1$. Here, $P_{i}$ is the transmit power of the user $i$, and $h_{j, i}$, for $i, j \in\{1,2\}$, is the Rayleigh channel coefficient between source $i$ and relay $j$ with i.i.d. circularly symmetric standard complex Gaussian entries, denoted by $h_{j, i} \sim \mathcal{C N}(0,1)$. The additive white Gaussian noise (AWGN) $n_{R_{j}}$ at relay $j$ is distributed according to $\mathcal{C N}(0,1)$. The average signal to noise ratio (SNR) of the source $i$ to the relay $j$ is $\gamma_{j, i}=P_{i}\left|h_{j, i}\right|^{2}$.

The relays decode the data from both of the users, reencode, and forward it to the destination at the second transmission hop. We only focus on one user's (e.g., user 1) data transmission from the relays to the destination ${ }^{3}$. Due to the symmetry, similar formulations can be derived for the other user. Let the decoded data of user 1 at relay 1 and relay 2 be denoted by $\hat{u}_{1, R_{1}}$ and $\hat{u}_{1, R_{2}}$, respectively. After reencoding at the relays, the corresponding coded and modulated versions are represented by $\hat{s}_{1, R_{1}}$ and $\hat{s}_{1, R_{2}}$, respectively, with $E\left\{\left|\hat{s}_{1, R_{1}}\right|^{2}\right\}=E\left\{\left|\hat{s}_{1, R_{2}}\right|^{2}\right\}=1$. Further assuming orthogonal

\footnotetext{
${ }^{1}$ Even though the wireless channels are static and stationary within a period of coherence time (or a block/frame/codeword length, equivalently), we only deliver symbol-wise formulations to all the received signal in this paper for the sake of simplicity.

${ }^{2}$ The encoding chain consists of source coding, channel coding, and modulation. The source coding rate of user $i$ is denoted by $R_{s_{i}}$ while the multiplication of channel coding rate and modulation order of user $i$ is denoted by $R_{c_{i}}$, for $i \in\{1,2\}$. The notations $R_{s_{i}}$ and $R_{c_{i}}$ are also applied to the relay $i$, for $i \in\{1,2\}$.

${ }^{3}$ All the outage analysis of the second hop, delivered in the Section III, is also based on user 1 .
}

transmission, e.g., time division multiple access (TDMA), the received data at the destination can be expressed as

$$
y_{D, t_{1}}=\sqrt{P_{R_{1}}} h_{1} \hat{s}_{1, R_{1}}+n_{D, t_{1}}
$$

and

$$
y_{D, t_{2}}=\sqrt{P_{R_{2}}} h_{2} \hat{s}_{1, R_{2}}+n_{D, t_{2}}
$$

where $n_{D, t_{1}}$ and $n_{D, t_{2}}$ denote the AWGN noise for two adjacent time indices $\left\{t_{1}, t_{2}\right\}$ with distribution $\mathcal{C N}(0,1)$. The relay 1 transmits with power $P_{R_{1}}$ while the relay 2 transmits with power $P_{R_{2}}$. The channel coefficient from relay $j$ to the destination is denoted by $h_{j}$ with $h_{j} \sim \mathcal{C N}(0,1)$, for $j \in\{1,2\}$. The average SNR of the relay $j$ to the destination is $\gamma_{j}=P_{R_{j}}\left|h_{j}\right|^{2}$, for $j \in\{1,2\}$.

\section{RATE REGION AND END-TO-END OUTAGE PROBABILITY}

The end-to-end outage probability is calculated based on the rate regions of two tandem transmissions, i.e., source-to-relay transmission and relay-to-destination transmission. Although we perform theoretical analysis for the two-source two-relay single-destination network, the extension to a generalized network is straightforward.

\section{A. Single-Antenna Relaying Systems}

The MAC rate region for sources-to-relay 1 at the first transmission hop is a bounded pentagonal region, shown in Fig. 2. A similar rate region can be achieved for sources-torelay 2 . We divide the rate region into four non-overlapping sub-regions as shown in Fig. 2. The rate pairs $\left(R_{c_{1}}, R_{c_{2}}\right)$ within $\mathcal{R}_{4}$ can guarantee error-free transmission of data from both user 1 and user 2 . On the contrary, any rate pairs $\left(R_{c_{1}}, R_{c_{2}}\right)$ within $\mathcal{R}_{3}$ lead to incorrect transmission of both users' data. Rate pairs $\left(R_{c_{1}}, R_{c_{2}}\right)$ within $\mathcal{R}_{1}$ or $\mathcal{R}_{2}$ can only ensure successful transmission of one user. Therefore, we can calculate the outage probabilities of the two users over the transmission links between the users and relay 1 .

The outage probabilities of user 1 and user 2 to relay 1 are [?]

$$
P_{\text {out }}^{1,1}=\operatorname{Pr}\left(\mathcal{R}_{1}\right)+\operatorname{Pr}\left(\mathcal{R}_{3}\right)
$$

and

$$
P_{\text {out }}^{1,2}=\operatorname{Pr}\left(\mathcal{R}_{2}\right)+\operatorname{Pr}\left(\mathcal{R}_{3}\right),
$$

which can be further calculated through two-fold integrals by taking into account the probability density functions (PDFs) of the two i.i.d. wireless propagation links.

The components $\operatorname{Pr}\left(\mathcal{R}_{1}\right)$ and $\operatorname{Pr}\left(\mathcal{R}_{3}\right)$ in (5) can be further expressed as

$$
\begin{array}{r}
\operatorname{Pr}\left(\mathcal{R}_{1}\right)=\operatorname{Pr}\left[R_{c_{1}}>\log _{2}\left(1+P_{1}\left|h_{1,1}\right|^{2}\right),\right. \\
\left.R_{c_{2}} \leq \log _{2}\left(1+\frac{P_{2}\left|h_{1,2}\right|^{2}}{1+P_{1}\left|h_{1,1}\right|^{2}}\right)\right],
\end{array}
$$

and

$$
\begin{array}{r}
\operatorname{Pr}\left(\mathcal{R}_{3}\right)=\operatorname{Pr}\left[R_{c_{1}}>\log _{2}\left(1+\frac{P_{1}\left|h_{1,1}\right|^{2}}{1+P_{2}\left|h_{1,2}\right|^{2}}\right),\right. \\
R_{c_{2}}>\log _{2}\left(1+\frac{P_{2}\left|h_{1,2}\right|^{2}}{1+P_{1}\left|h_{1,1}\right|^{2}}\right), \\
\left.R_{c_{1}}+R_{c_{2}}>\log _{2}\left(1+P_{1}\left|h_{1,1}\right|^{2}+P_{2}\left|h_{1,2}\right|^{2}\right)\right] .
\end{array}
$$




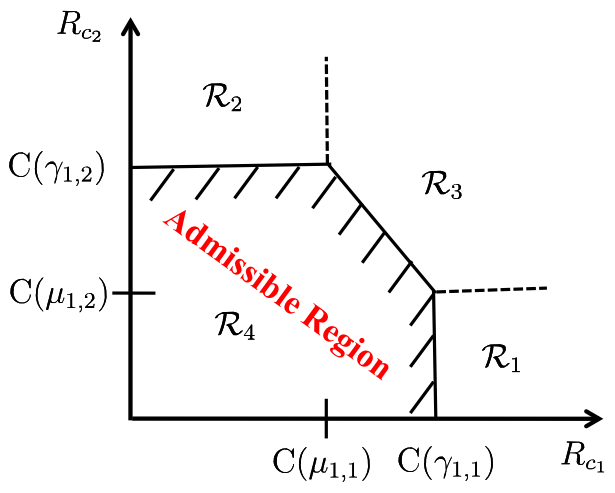

Fig. 2: Rate region for sources-to-relay 1 transmission links divided into four non-intersecting sub-regions. Here, $\mu_{1,1}=P_{1}\left|h_{1,1}\right|^{2} /(1+$ $\left.P_{2}\left|h_{1,2}\right|^{2}\right)$ and $\mu_{1,2}=P_{2}\left|h_{1,2}\right|^{2} /\left(1+P_{1}\left|h_{1,1}\right|^{2}\right)$ denote the signal to interference plus noise ratio of user 1 and user 2 , respectively. The expression $\mathrm{C}(a)=\log _{2}(1+a)$ denotes the capacity calculation function.

Setting $\left|h_{1,1}\right|^{2}=H_{1}$ and $\left|h_{1,2}\right|^{2}=H_{2}$, it is not difficult to get that $H_{1}$ and $H_{2}$ are i.i.d. exponential distribution random variables with unit mean. Furthermore, we assume that equal power is allocated to both of the users, i.e., $P_{1}=P_{2}=P$. Then, (7) and (8) can be further expressed as

$$
\begin{aligned}
\operatorname{Pr}\left(\mathcal{R}_{1}\right) & =\int_{H_{1}=0}^{\frac{2^{R_{c_{1}-1}}}{P}} \exp \left(-H_{1}\right) d H_{1} \\
& \int_{H_{2}=\frac{\left(2^{\left.R_{c_{2}}-1\right)\left(1+P H_{1}\right)}\right.}{P}}^{\infty} \exp \left(-H_{2}\right) d H_{2} \\
& =\frac{1}{2^{R_{c_{2}}}}\left[\exp \left(-\frac{2^{R_{c_{2}}}-1}{P}\right)-\exp \left(-\frac{2^{R_{c_{1}}+R_{c_{2}}-1}}{P}\right)\right],
\end{aligned}
$$

and

$$
\begin{aligned}
& \operatorname{Pr}\left(\mathcal{R}_{3}\right)=\int_{H_{1}=0}^{\frac{2^{R_{c_{1}}-1}}{P}} \int_{H_{2}=0}^{\frac{\left(2^{\left.R_{c_{2}}-1\right)\left(1+P H_{1}\right)}\right.}{P}} \exp \left(-H_{1}-H_{2}\right) d H_{1} d H_{2} \\
& +\int_{H_{1}=\frac{2^{R_{c_{1}}-1}}{P}}^{\frac{2^{\left.R_{c_{2}(2} R_{c_{1}}-1\right)}}{P}} \exp \left(-H_{1}\right) d H_{1} \\
& \int_{H_{2}=\frac{H_{1}}{2^{R_{c_{1}}-1}}-\frac{1}{P}}^{P} \exp \left(-H_{2}\right) d H_{2} \\
& =1-\frac{1}{2^{R_{c_{1}}}} \exp \left(-\frac{2^{R_{c_{1}}}-1}{P}\right)-\frac{1}{2^{R_{c_{2}}}} \exp \left(-\frac{2^{R_{c_{2}}}-1}{P}\right) \\
& -\exp \left(-\frac{2^{R_{c_{1}}+R_{c_{2}}}-1}{P}\right)\left[1-\frac{1}{2^{R_{c_{1}}}}-\frac{1}{2^{R_{c_{2}}}}\right] \\
& -\exp \left(-\frac{2^{R_{c_{1}}+R_{c_{2}}}-1}{P}\right) \frac{\left(2^{R_{c_{1}}}-1\right)\left(2^{R_{c_{2}}}-1\right)}{P} .
\end{aligned}
$$

The probability (also called occurrence probability) of errorfree decoding of user 1 's data at the relay 1 is $1-P_{\text {out }}^{1,1}$. According to (6), the outage probability of user 2 at relay 1 $\left(P_{\text {out }}^{1,2}\right)$ can be derived by following similar calculations shown in (7)-(10). Similarly, the outage probabilities $\left\{P_{\text {out }}^{2,1}, P_{\text {out }}^{2,2}\right\}^{4}$ of transmission links from the users to relay 2 can be calculated based on their corresponding MAC rate region.

\footnotetext{
${ }^{4} \mathrm{Here}, P_{\mathrm{out}}^{2,1}$ denotes the outage probability of user 1 at relay 2 while $P_{\text {out }}^{2,2}$ denotes the outage probability of user 2 at relay 2 .
}

Based on the outcome of data recovery of user 1 at the relays, we divide the analysis into three cases as shown in Table I. In the following, we give further details of these cases.

Case $1\left(\hat{u}_{1, R_{1}} \neq u_{1}, \hat{u}_{1, R_{2}} \neq u_{1}\right)$ : In this case, both of the relays cannot fully recover the data from user 1 . Therefore, the two decoded data at the relays can be regarded as noisy versions of the original data. Thus, the problem falls into the category of chief executive officer (CEO) problem at the second hop (i.e., relay-to-destination transmission). Even though the performance with respect to outage probabilities can be improved by partial hybrid automatic repeat request (HARQ) technique [?], here, for the purpose of simplicity, we just set the outage probability to 1 at the second transmission.

Case $2\left(\hat{u}_{1, R_{1}}=u_{1}, \hat{u}_{1, R_{2}}=u_{1}\right)$ : In this case, we break the assumption of orthogonal transmission made in Section II. Nonorthogonal transmission is assumed and maximum ratio transmission $^{5}$ (MRT) technique is applied to achieve the maximum diversity gain (second order). Then, the outage probability can be obtained through two-fold integral computation.

Assuming $P_{R_{2}}=P_{R_{2}}=P$, the received signal at the destination can be reformulated as ${ }^{6}$

$$
\begin{aligned}
y_{D} & =\frac{\sqrt{P} h_{1} h_{1}^{*}}{\sqrt{\left|h_{1}\right|^{2}+\left|h_{2}\right|^{2}}} s_{1}+\frac{\sqrt{P} h_{2} h_{2}^{*}}{\sqrt{\left|h_{1}\right|^{2}+\left|h_{2}\right|^{2}}} s_{1}+n \\
& =\sqrt{P} \sqrt{\left|h_{1}\right|^{2}+\left|h_{2}\right|^{2}} s_{1}+n,
\end{aligned}
$$

where $(\cdot)^{*}$ denotes the operation of complex conjugate, and $n$ is the complex normal distributed AWGN noise.

Setting $\left|h_{1}\right|^{2}=H 3$ and $\left|h_{2}\right|^{2}=H 4$, and assuming both of the relays transmit with the same rate $R_{c}$, the outage probability can be expressed as

$$
\begin{aligned}
P_{\text {out }}(\text { Case 2) } & =\operatorname{Pr}\left\{R_{c}>\log _{2}\left(1+P\left(\left|h_{1}\right|^{2}+\left|h_{2}\right|^{2}\right)\right)\right\} \\
& =\int_{0}^{\frac{2^{R_{c}-1}}{P}} \int_{0}^{\frac{2^{R_{c}-1}}{P}-H_{3}} \exp \left(-H_{3}-H_{4}\right) d H_{3} d H_{4} \\
& =1-\exp \left(-\frac{2^{R_{c}}-1}{P}\right)\left(1+\frac{2^{R_{c}}-1}{P}\right) .
\end{aligned}
$$

Alternatively, the outage probability for the Case 2 can be calculated by considering the two relay-to-destination transmission links separately. The outage occurs when neither data sequence from the relays is successfully recovered at the destination.

Case $3\left(\hat{u}_{1, R_{1}} \neq u_{1}, \hat{u}_{1, R_{2}}=u_{1}\right)$ or $\left(\hat{u}_{1, R_{1}}=u_{1}, \hat{u}_{1, R_{2}} \neq\right.$ $\left.u_{1}\right)$ : For this scenario, only one of the relays, either relay 1 or relay 2 , can successfully decode the original data depending on their admissible rate regions. The erroneously decoded message at one relay is correlated with the source data. In addition, the fully recovered message at the other relay is exactly the same with the source sequence. Therefore, the decoded sequences at the relays are correlated, entitled as relay-relay correlation. In this regards, the problem turns into the category of source coding with helper.

In order to simplify the problem of calculating outage probability at the second hop, we exploit bit flipping model

\footnotetext{
${ }^{5}$ In order to implement MRT, global channel state information is assumed to be available at the transmitter side.

${ }^{6}$ Both of the relays can successfully recover the data from user 1 , thus the encoded data at the relays is the same with that originated from user 1 assuming the same coding chain is applied to both user 1 and relays.
} 
TABLE I: THREE POSSIBLE CASES/SCENARIOS OF USER 1'S DECODED DATA AT THE TWO RELAY NODES

\begin{tabular}{|c|c|c|c|}
\hline & Case 1 & Case 2 & Case 3 \\
\hline Scenarios & $\left(\hat{u}_{1, R_{1}} \neq u_{1}, \hat{u}_{1, R_{2}} \neq u_{1}\right)$ & $\left(\hat{u}_{1, R_{1}}=u_{1}, \hat{u}_{1, R_{2}}=u_{1}\right)$ & $\left(\hat{u}_{1, R_{1}} \neq u_{1}, \hat{u}_{1, R_{2}}=u_{1}\right)$ or $\left(\hat{u}_{1, R_{1}}=u_{1}, \hat{u}_{1, R_{2}} \neq u_{1}\right)$ \\
\hline Occurrence Probabilities & $P_{\text {out }}^{1,1} P_{\text {out }}^{2,1}$ & $\left(1-P_{\text {out }}^{1,1}\right)\left(1-P_{\text {out }}^{2,1}\right)$ & $P_{\text {out }}^{1,1}\left(1-P_{\text {out }}^{2,1}\right)+\left(1-P_{\text {out }}^{1,1}\right) P_{\text {out }}^{2,1}$ \\
\hline
\end{tabular}

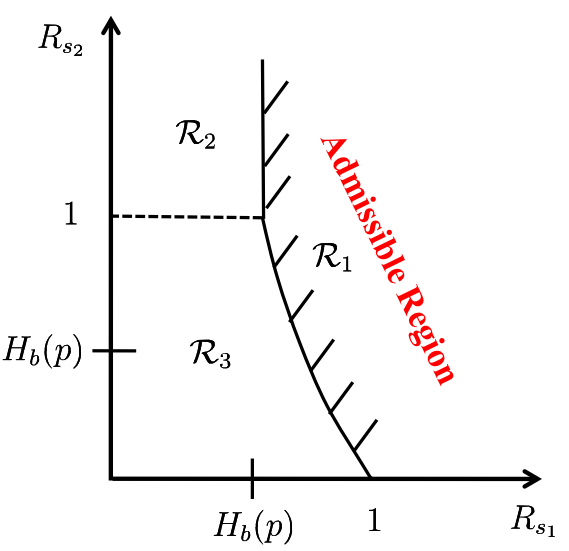

Fig. 3: The admissible rate region determined by source coding with helper theorem.

to model the correlation between the decoded data at the two relays, e.g., $\hat{u}_{1, R_{1}}=\hat{u}_{1, R_{2}} \oplus e=u_{1} \oplus e$ for the first sub-case of Case 3 , where $\operatorname{Pr}\{e=1\}=p$ is the relay-relay correlation coefficient and $\oplus$ denotes modulo- 2 addition. According to the lossy source channel separation theorem, the inequality $R_{s_{1}}(D) R_{c_{1}} \leq \mathrm{C}\left(\gamma_{j, 1}\right)$ holds at the first hop by assuming that the interference from user 2 are successfully canceled, for $j \in\{1,2\}$, where $D$ is the distortion measurement, e.g., Hamming distance/error probability for binary sources. In this regard, we can calculate the relay-relay correlation $p$ by setting $R_{s_{1}}(p) R_{c_{1}}=\mathrm{C}\left(\gamma_{j, 1}\right)$, for $j \in\{1,2\}$. The outage problem at the second hop turns into source coding with helper, where the achievable rate region is shown in Fig. 3. It can be expressed as $^{7}$

$$
R_{s_{1}} \geq \begin{cases}H_{b}(p), & \text { for } R_{s_{2}} \geq 1 \\ H_{b}(\alpha * p), & \text { for } 0 \leq R_{s_{2}} \leq 1\end{cases}
$$

where $\alpha$ is the relay-destination correlation, i.e., $\alpha=$ $\operatorname{Pr}\left(\hat{u}_{1, R_{j}} \neq \hat{u}_{1, D}\right)$ for $j \in\{1,2\}$ with $\hat{u}_{1, D}$ being the decoded data at the destination, and $*$ represents convolution operation, e.g., $a * b=a(1-b)+(1-a) b$ with $0 \leq a, b \leq 1$.

The outage probability of Case 3 can be expressed as

$$
P_{\text {out }}(\text { Case } 3)=\operatorname{Pr}\left(\mathcal{R}_{2}\right)+\operatorname{Pr}\left(\mathcal{R}_{3}\right) \text {. }
$$

Moreover, regarding the first sub-case of Case 3, we have the following two equalities according to the lossless and lossy source channel separation theorems.

$$
\begin{aligned}
& R_{s_{1}}(\alpha) R_{c_{1}}=\mathrm{C}\left(\gamma_{1}\right), \\
& R_{s_{2}} R_{c_{2}}=\mathrm{C}\left(\gamma_{2}\right),
\end{aligned}
$$

where $\gamma_{1}=P H_{3}, \gamma_{2}=P H_{4}$ assuming $P_{R_{1}}=P_{R_{2}}=P$. Similar equalities can be obtained for the second sub-case of Case 3.

\footnotetext{
${ }^{7} H_{b}(\cdot)$ denotes the binary entropy function, i.e., $H_{b}(p)=-p \log (p)-$ $(1-p) \log (1-p)$.
}

Then, the probabilities $\operatorname{Pr}\left(\mathcal{R}_{2}\right)$ and $\operatorname{Pr}\left(\mathcal{R}_{3}\right)$ in (14) can further be written by ${ }^{8}$

$$
\begin{aligned}
& \operatorname{Pr}\left(\mathcal{R}_{2}\right)=\operatorname{Pr}\left(R_{s_{2}} \geq 1,0 \leq R_{s_{1}} \leq H_{b}(p)\right) \\
& =\int_{\mathrm{C}^{-1}\left(R_{c_{2}}\right) / P}^{\infty} \int_{0}^{\mathrm{C}^{-1}\left(R_{c_{1}} H_{b}(p)\right) / P} \exp \left(-H_{3}-H_{4}\right) d H_{3} d H_{4},
\end{aligned}
$$

and

$$
\begin{aligned}
& \operatorname{Pr}\left(\mathcal{R}_{3}\right)=\operatorname{Pr}\left(0 \leq R_{s_{2}} \leq 1,0 \leq R_{s_{1}} \leq H_{b}(\alpha * p)\right) \\
& =\int_{0}^{\mathrm{C}^{-1}\left(R_{c_{2}}\right) / P} \int_{0}^{\mathrm{C}^{-1}\left(R_{c_{1}} H_{b}(\alpha * p)\right) / P} \exp \left(-H_{3}-H_{4}\right) d H_{3} d H_{4} .
\end{aligned}
$$

The overall end-to-end outage probability of user 1 can be expressed as

$$
\begin{aligned}
& P_{\text {out }}^{E 2 E}=P_{\text {out }}^{1,1} P_{\text {out }}^{2,1}+\left(1-P_{\text {out }}^{1,1}\right)\left(1-P_{\text {out }}^{2,1}\right) P_{\text {out }}(\text { Case } 2) \\
& +\left(P_{\text {out }}^{1,1}\left(1-P_{\text {out }}^{2,1}\right)+\left(1-P_{\text {out }}^{1,1}\right) P_{\text {out }}^{2,1}\right) P_{\text {out }}(\text { Case } 3) .
\end{aligned}
$$

\section{B. Multiple-Antenna Relaying Systems}

It is of great challenge to find an optimal coding scheme that achieves the outer bound of the MAC rate region if the relays are equipped with a single antenna. The Kasami codes presented in [?] are suboptimal, which can only achieve a point between the inner bound and outer bound of the rate region. To simplify the code design process, we introduce the concept of multiple-input multiple-output (MIMO) into the MAC channel, which can easily convert the MAC transmission into a point-to-point transmission by virtue of uncomplicated algebraic manipulations, for example, matrix inverse, singular value decomposition (SVD) [?] at the relay nodes. As a result, the rate region is changed from a bounded pentagon into a bounded rectangle.

We further assume that the number of the antennas at each relay is equal to the number of users to simplify the problem. In our proposed topology, we assume that two antennas are equipped at each of the relay nodes. Therefore, by postulating equal power transmission, the received signal at the relay 1 can be expressed as

$$
\mathbf{y}=\sqrt{P} \mathbf{H s}+\mathbf{n},
$$

where $\mathbf{H} \in \mathbb{C}^{2 \times 2}$ is the channel coefficient matrix with each entry i.i.d. normal Gaussian distributed, $\mathbf{s}=\left[\begin{array}{ll}s_{1} & s_{2}\end{array}\right]^{T}$, and $\mathbf{n} \in \mathbb{C}^{2 \times 1}$ is Gaussian vector with each component normal Gaussian distributed.

We assume that the channel state information (CSI) is available at the relay side. By simply applying ZF filtering [?] at the relay node, the post-detected signal can be written by

$$
\hat{\mathbf{s}}=\left(\mathbf{H}^{H} \mathbf{H}\right)^{-1} \mathbf{H}^{H} \mathbf{y}=\sqrt{P} \mathbf{s}+\mathbf{H}^{-1} \mathbf{n},
$$

\footnotetext{
${ }^{8}$ The notation $\mathrm{C}^{-1}(\cdot)$ in (16) and (17) denotes the inversion function of the capacity calculation function. More information about the calculation of $\operatorname{Pr}\left(\mathcal{R}_{2}\right)$ and $\operatorname{Pr}\left(\mathcal{R}_{3}\right)$ can be find in [?].
} 


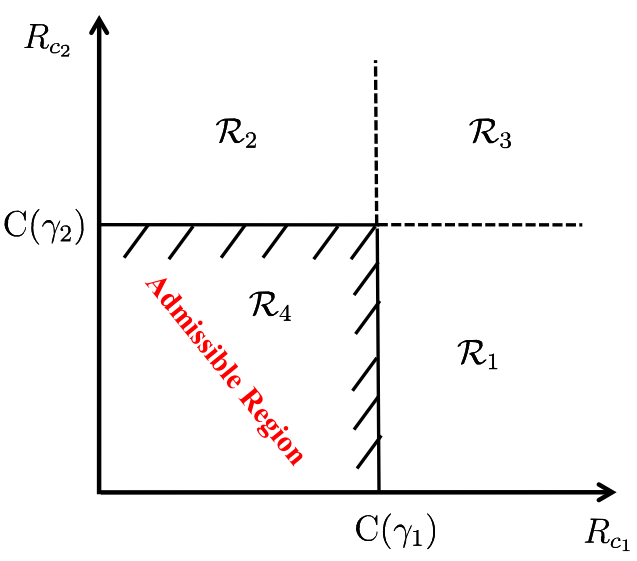

Fig. 4: Rate region of multiple-antenna relaying system for the source-to-relay transmission.

where $(\cdot)^{H}$ and $(\cdot)^{-1}$ stand for conjugate transpose and inverse, respectively.

By using multiple antennas at the relay nodes, the multiple access transmission is transformed to a point-to-point transmission. Accordingly, the rate region becomes rectangular, as shown in Fig. 4.

The singular value decomposition (SVD) is applied to decompose $\mathbf{H}=\mathbf{U} \Sigma \mathbf{V}^{H}$, where $\mathbf{U} \in \mathbb{C}^{2 \times 2}$ and $\mathbf{V} \in \mathbb{C}^{2 \times 2}$ denote the left and right singular (and unitary) matrices of $\mathbf{H}$, respectively. Here, $\Sigma \in \mathbb{C}^{2 \times 2}$ is a diagonal matrix $\Sigma=\operatorname{diag}\left(\left[\sigma_{1}, \sigma_{2}\right]\right)$ with $\sigma_{1} \geq \sigma_{2} \geq 0$. The post-detection SNR for user $i$ can be written as ${ }^{9}$

$$
\gamma_{i}=P \sigma_{i}^{2} .
$$

We set $\lambda_{i}=\sigma_{i}^{2}$, for $i \in\{1,2\}$, which are eigenvalues of covariance matrix of $\mathbf{H}$ and mutually dependent. The joint PDF for the $\lambda_{i}$ 's can be expressed as [?]

$$
p\left(\lambda_{1}, \lambda_{2}\right)=K \exp \left(-\lambda_{1}-\lambda_{2}\right)\left(\lambda_{1}-\lambda_{2}\right)^{2},
$$

where $K$ is a constant normalization factor ensuring that the $\int_{0}^{\infty} \int_{0}^{\infty} p\left(\lambda_{1}, \lambda_{2}\right) d \lambda_{1} d \lambda_{2}=1$. The resultant value for $K$ is 0.5 . According to (22), we can calculate the marginal PDF for each $\lambda_{i}$ and the result can be expressed as

$$
p\left(\lambda_{i}\right)=\frac{1}{2} \exp \left(-\lambda_{i}\right)\left(\lambda_{i}^{2}-2 \lambda_{i}+2\right), \text { for } i=1,2 .
$$

The derived theoretical PDF of $\lambda_{i}$ in (23) is verified by MonteCarlo simulations, which is shown in Fig. 5. The MonteCarlo simulation result almost overlaps with the its theoretical counterpart, except for the two tiny intervals $[0,0.1]$ and $[9.8,10]$. This tiny mismatch can further be improved by using sufficient measurement data. Following the PDF from (23), the outage probability of user $i$ at relay 1 can be further calculated by

$$
\begin{aligned}
P_{\text {out }}^{1, i} & =\operatorname{Pr}\left(\gamma_{i}<2^{R_{c_{i}}}-1\right)=\int_{0}^{\frac{2^{R_{c_{i}}-1}}{P}} p\left(\lambda_{i}\right) d \lambda_{i} \\
& =1-\frac{\exp \left(-\frac{2^{R_{c_{i}}-1}}{P}\right)\left(\left(\frac{2^{R_{c_{i}}-1}}{P}\right)^{2}+2\right)}{2} .
\end{aligned}
$$

${ }^{9}$ Note that the abuse of notation happens for $\gamma_{i}$ 's here.

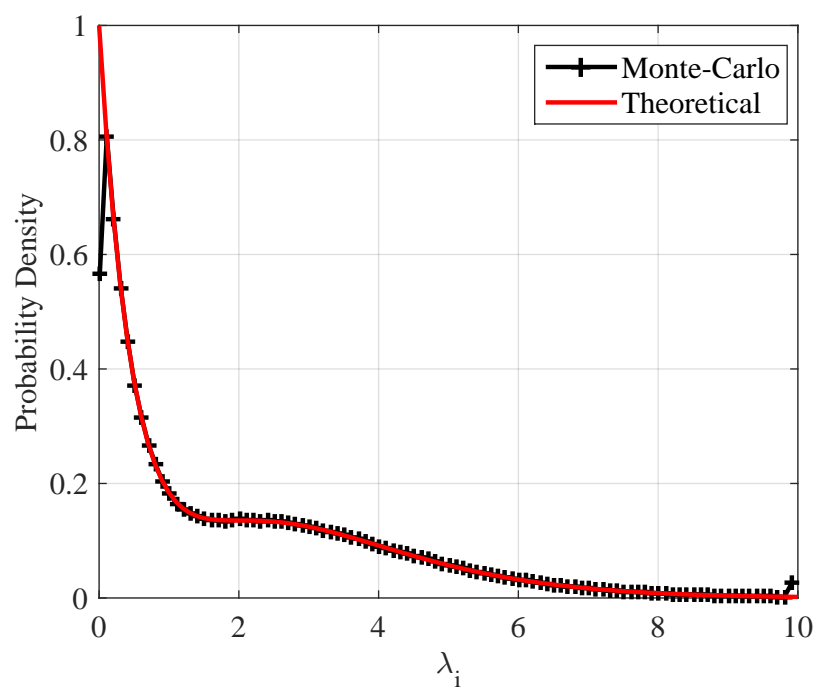

Fig. 5: Theoretical PDF of $\lambda_{i}$ from (23) and numerical PDF of $\lambda_{i}$ through Monte-Carlo simulations.

Case $1\left(\hat{u}_{1, R_{1}} \neq u_{1}, \hat{u}_{1, R_{2}} \neq u_{1}\right)$ : In this case, we follow the same rule as for single-antenna relaying system by setting the outage probability of second hop to 1 .

Case $2\left(\hat{u}_{1, R_{1}}=u_{1}, \hat{u}_{1, R_{2}}=u_{1}\right)$ : Since both of the relays have two antennas. Hence the channels between relays and destination are supposed to be $\mathbf{h}_{1} \in \mathbb{C}^{1 \times 2}$ and $\mathbf{h}_{2} \in \mathbb{C}^{1 \times 2}$. By using MRT, the received signal can be expressed as

$$
\begin{aligned}
y & =\frac{\sqrt{P} \mathbf{h}_{1} \mathbf{h}_{1}^{H}}{\sqrt{\left\|\mathbf{h}_{1}\right\|_{2}^{2}+\left\|\mathbf{h}_{2}\right\|_{2}^{2}}} s_{1}+\frac{\sqrt{P} \mathbf{h}_{2} \mathbf{h}_{2}^{H}}{\sqrt{\left\|\mathbf{h}_{1}\right\|_{2}^{2}+\left\|\mathbf{h}_{2}\right\|_{2}^{2}}} s_{1}+n, \\
& =\sqrt{P} \sqrt{\mathbf{h}_{1} \mathbf{h}_{1}^{H}+\mathbf{h}_{2} \mathbf{h}_{2}^{H}} s_{1}+n,
\end{aligned}
$$

where $\|\cdot\|_{2}$ denotes the vector two-norm. By an abuse of notations $\left\{H_{3}, H_{4}\right\}$ and setting $\mathbf{h}_{1} \mathbf{h}_{1}^{H}=H_{3}$ and $\mathbf{h}_{2} \mathbf{h}_{2}^{H}=H_{4}$, $H_{3}$ and $H_{4}$ are Chi-square random variables. The PDF of $H_{i}$, for $i \in\{3,4\}$ can be expressed as

$$
p\left(H_{i}\right)=H_{i} \exp \left(-H_{i}\right) .
$$

The corresponding outage probability can be computed by

$$
P_{\text {out }}(\text { Case } 2)=\operatorname{Pr}\left(R_{c}>\log _{2}\left(1+P\left(H_{3}+H_{4}\right)\right)\right) .
$$

Then, the final expression for (27) can be written by

$$
\begin{aligned}
& \operatorname{Pr}\left\{R_{c}>\log _{2}\left(1+P\left(H_{3}+H_{4}\right)\right)\right\} \\
& =\int_{0}^{\frac{2^{R_{c}-1}}{P}} \int_{0}^{\frac{2^{R_{c}-1}}{P}-H_{3}} H_{3} H_{4} \exp \left(-H_{3}-H_{4}\right) d H_{3} d H_{4} \\
& =1-\exp \left(-\frac{2^{R_{c}}-1}{P}\right)\left(1+\frac{2^{R_{c}}-1}{P}+\frac{\left(2^{R_{c}}-1\right)^{2}}{2 P^{2}}+\frac{\left(2^{R_{c}}-1\right)^{3}}{6 P^{3}}\right) .
\end{aligned}
$$

Case $3\left(\hat{u}_{1, R_{1}} \neq u_{1}, \hat{u}_{1, R_{2}}=u_{1}\right)$ or $\left(\hat{u}_{1, R_{1}}=u_{1}, \hat{u}_{1, R_{2}} \neq\right.$ $\left.u_{1}\right)$ : In this case, we again utilize source coding with helper theorem, as already applied to single-antenna relaying systems, 


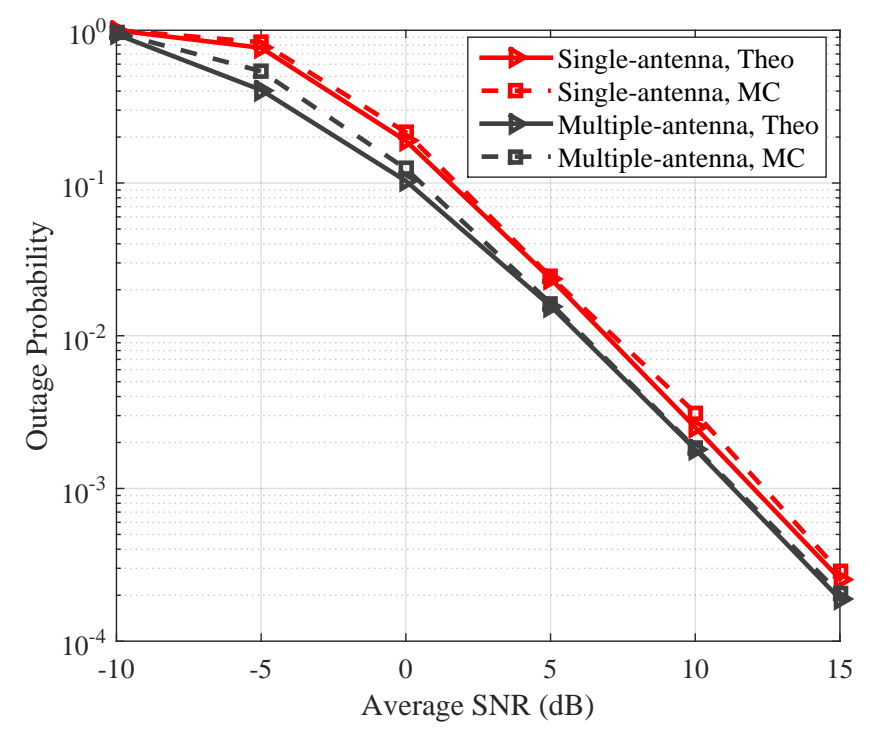

Fig. 6: End-to-end outage probabilities of user 1 in the multi-source multi-relay system.

to compute the two components (referring to (14)) of the outage probability.

$\operatorname{Pr}\left(\mathcal{R}_{2}\right)=\operatorname{Pr}\left(R_{s_{2}} \geq 1,0 \leq R_{s_{1}} \leq H_{b}(p)\right)$
$=\int_{\mathrm{C}^{-1}\left(R_{c_{2}}\right) / P}^{\infty} \int_{0}^{\mathrm{C}^{-1}\left(R_{c_{1}} H_{b}(p)\right) / P} H_{3} H_{4} \exp \left(-H_{3}-H_{4}\right) d H_{3} d H_{4}$,

and

$\operatorname{Pr}\left(\mathcal{R}_{3}\right)=\operatorname{Pr}\left(0 \leq R_{s_{2}} \leq 1,0 \leq R_{s_{1}} \leq H_{b}(\alpha * p)\right)$
$=\int_{0}^{\mathrm{C}^{-1}\left(R_{c_{2}}\right) / P} \int_{0}^{\mathrm{C}^{-1}\left(R_{c_{1}} H_{b}(\alpha * p)\right) / P} H_{3} H_{4} \exp \left(-H_{3}-H_{4}\right) d H_{3} d H_{4}$.

\section{NumericAl RESUlts}

In this section, we provide Monte-Carlo simulations to calculate the end-to-end outage probabilities (only for user 1, the same results can be obtained for user 2 due to the symmetry of the proposed topology) and compare them with the derived theoretical results. Moreover, we compare the single-antenna relaying systems and the multiple-antenna relaying systems. For all the transmission links, we use the same transmit powers and multiplications of channel coding rate and modulation order. All the rates, including $R_{c_{1}}, R_{c_{2}}$ and $R_{c}$, are set to be 0.5 .

We can clearly observe from the results presented in Fig. 6 that our numerical examples closely approach the theoretical analysis. It is depicted in Fig. 6 that the second order diversity is achieved for both relaying systems in terms of end-to-end outage probabilities due to the two independent routes from each source to the destination. The multiple-antenna relaying system slightly outperforms its single-antenna counterpart in terms of outage probability. The reason for this lies in the fact that multiplexing gain is exploited at the first hop and meanwhile second order diversity gain can only be obtained at the second hop for the multiple-antenna relaying systems.
Most importantly, the multiple-antenna relaying systems can readily solve the issue of practical and optimal code design.

\section{COnClusion And Future Work}

We have analyzed the end-to-end outage probability for the multi-source multi-relay network. By employing the twohop transmission, we have calculated the end-to-end outage probability by considering the occurrence probabilities in the first hop and the outage probabilities in the second hop. Both the single-antenna and multiple-antenna relaying systems have been investigated and subsequently compared. The theoretical analysis has demonstrated the improved performance of the multiple-antenna relaying systems as compared to the singleantenna relaying systems. The theoretical analysis has been verified by the numerical simulations and it has been shown that the numerical examples closely follow the theoretical analysis. In addition to improved performance, the introduction of MIMO concept to the multi-source multi-relay network also solves the problem of practical code design.

The exact outage probability calculation of CEO case at the second hop is a potential future research topic. For further improving outage performance, optimal power allocation at the sources and relays can be investigated. For the single-antenna relaying systems, practical code design and joint decoding algorithm by taking into account the correlation information could lead to another potential future research topic. 